\title{
Construcción de tablas estadísticas por estudiantes chilenos de tercero de Educación Primaría
}

\author{
Danilo Díaz-Levicoy* \\ Rodolfo Morales ${ }^{* *}$ \\ Claudia Vásquez Ortiz***
}

\section{Resumen}

En el presente artículo reportamos los resultados sobre la construcción de tablas estadísticas por grupo de estudiantes de tercer grado de Educación Primaria en Chile. La muestra estuvo compuesta por 79 estudiantes, a los que se les aplicó un cuestionario con dos actividades, previamente validado por expertos. En la primera actividad se solicitó a los estudiantes construir una tabla de conteo a partir de un listado de datos sin agrupar, mientras que en la segunda se solicitó construir una tabla a partir de la información de un pictograma. Los resultados muestran que la actividad en la que el estudiante debe completar la tabla de conteo y obtener la frecuencia con ellos es la más sencilla, mientras que en la que debe completarla de acuerdo con la información de un pictograma les resulta más difícil ya que no consideran que el icono no tenga valor unitario.

Palabras clave: construcción, tablas estadísticas, Educación Primaria.

* Máster en Didáctica de la Matemática (Universidad de Granada). Doctorando en Ciencias de la Educación en la Universidad de Granada, España.

** Máster en Didáctica de la Matemática (Universidad de Granada). Doctorando en Ciencias de la Educación en la Universidad de Granada, España.

*** Doctora en Educación (Universidad de Gerona). Profesora Asistente de la Pontificia Universidad Católica de Chile, Chile. 


\section{Construction of statistical tables by Chilean students of third grade of Primary Education}

\section{Abstract}

The present study reports the results on the construction of statistical tables by group of third grade Primary Education students in Chile. The sample consisted of 79 students to whom a questionnaire with two activities, previously validated by experts, was applied. In the first activity students were asked to make a counting table from a list of ungrouped data, whereas in the second one, a table was asked to build from the information in a pictogram. Results show that the activity in which the students must complete the counting table and get the frequency with them is simpler, while the one in which they must complete according to the information of a pictogram is more difficult for the students since they do not consider that the icon has no unitary value.

Keywords: construction, statistical tables, Primary Education.

\section{Construção de tabelas estatísticas por estudantes chilenos de terceira ano do Ensino Fundamental}

\section{Resumo}

Neste artigo relatamos os resultados sobre a construção de tabelas estatísticas por grupo de alunos do terceiro ano do Ensino Fundamental no Chile. A amostra foi composta por 79 estudantes, aos quais foi aplicado um questionário com duas atividades, previamente validados por especialistas. $\mathrm{Na}$ primeira atividade, os estudantes foram solicitados a construir uma tabela de contagem a partir de uma lista de dados não agrupados, enquanto na segunda, uma tabela foi solicitada a ser compilada a partir das informações em um pictograma. Os resultados mostram que a atividade em que o aluno deve completar a tabela de contagem e obter a frequência com eles é a mais simples, enquanto em que eles devem completá-lo de acordo com as informações de um pictograma, é mais difícil para eles, porque consideram que o ícone não tem valor unitário.

Palavras-chave: construção, tabelas estatísticas, ensino primário.

\section{Introducción}

Diferentes países han incorporado la enseñanza de la estadística y la probabilidad desde los primeros cursos de la enseñanza 
obligatoria (e.g., EEUU, España, Brasil y Chile). Esto motivado por la importancia de esta disciplina en diferentes ámbitos de la vida cotidiana, por ejemplo, en los noticiarios se puede observar gráficos estadísticos para mostrar la cantidad de desempleo en los últimos meses, una tabla con la cantidad de goles de cada equipo de fútbol que participan en una competencia, entre otras situaciones.

La importancia social y estadística de estas representaciones, y en particular de las tablas estadísticas, las hacen un componte de la cultura estadística (CAZORLA; UTSUMI, 2010; MURRAY; GAL, 2002; GAL, 2002; WALLMAN, 1993; WATSON, 1997), término que surge de forma espontánea entre estadísticos y educadores estadísticos (BATANERO, 2004) y que hace referencia a dos habilidades:

a) Interpretar y evaluar críticamente la información estadística, los argumentos apoyados en datos o los fenómenos estocásticos que las personas pueden encontrar en diversos contextos, y b) discutir o comunicar sus opiniones respecto a tales informaciones estadísticas cuando sea relevante (GAL, 2002, p. 2-3).

En el contexto chileno, las últimas modificaciones a las directrices curriculares incorporan el estudio de la estadística y la probabilidad de forma continua y gradual a lo largo de todo el currículo escolar (Figura 1), con lo que se busca dar respuesta a las distintas necesidades presentes en cada una de las distintas etapas de la formación escolar.

Considerando la enseñanza de las tablas estadísticas desde la Educación Primaria (MINISTERIO DE EDUCACIÓN, 2012), donde el trabajo en los dos primeros años se basan en la lectura y construcción de tablas de conteo ya sea con datos provenientes de contextos cercamos al estudiante, o bien a partir del registro de datos a partir de juegos con de dados y monedas (MINISTERIO DE EDUCACIÓN, 2013a, 2013b) y que se observa en el Cuadro 1. 
Figura 1. Atención de los temas de estadística y probabilidad en el currículo de enseñanza obligatoria en Chile (VÁSQUEZ, 2018).

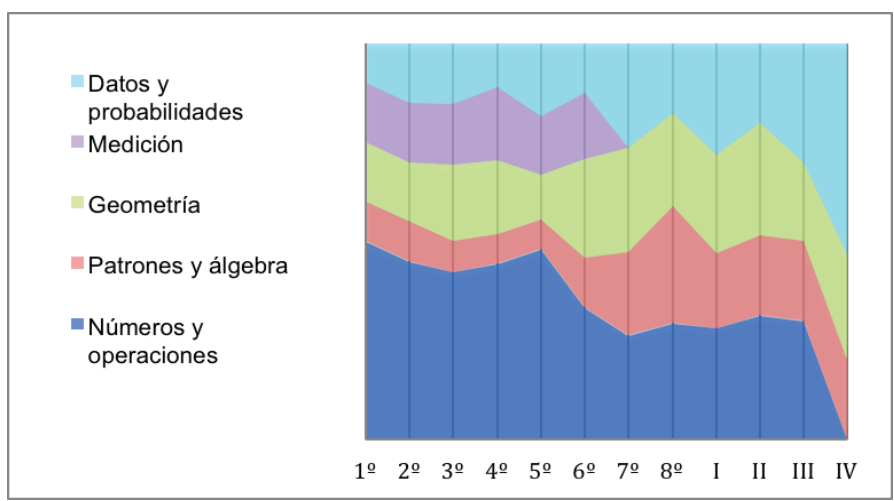

Cuadro 1. Objetivos e indicadores de evaluación para $1^{\circ}$ y $2^{\circ}$ de Educación Primaria

\begin{tabular}{|c|c|c|}
\hline Curso & Objetivo & Indicador de evaluación \\
\hline Primero & Recolectar y registrar & - Recolectan y organizan datos del \\
\hline (MINISTERIO & datos para responder & entorno, usando material concreto y \\
\hline DE & preguntas estadísticas & pictórico, registros informales y tablas \\
\hline EDUCACIÓN, & sobre sí mismo y & de conteo. \\
\hline 2013a, p. 105) & el entorno, usando & - Responden preguntas, utilizando la \\
\hline & $\begin{array}{l}\text { bloques, tablas de conteo } \\
\text { y pictogramas. }\end{array}$ & colectada. \\
\hline \multirow{7}{*}{$\begin{array}{l}\text { Segundo } \\
\text { (MINISTERIO } \\
\text { DE } \\
\text { EDUCACIÓN, } \\
2013 \text { b, p. } 116, \\
136)\end{array}$} & $\begin{array}{l}\text { Recolectar y registrar } \\
\text { datos para responder }\end{array}$ & $\begin{array}{l}\text { - Recolectan datos acerca de lanzamientos } \\
\text { de dados y monedas. }\end{array}$ \\
\hline & preguntas estadísticas & - Registran datos en una tabla de conteo \\
\hline & sobre juegos con & acerca de datos de lanzamientos de \\
\hline & monedas y dados, & monedas y dados. \\
\hline & $\begin{array}{l}\text { usando bloques, tablas } \\
\text { de conteo y pictogramas. }\end{array}$ & $\begin{array}{l}\text { Registran datos acerca de lanzamientos } \\
\text { de dados y monedas, usando cubos } \\
\text { apilables. }\end{array}$ \\
\hline & & $\begin{array}{l}\text { - Responden preguntas en el contexto de } \\
\text { juegos con monedas, usando registros } \\
\text { expresados en cubos apilables. }\end{array}$ \\
\hline & $\begin{array}{l}\text { Registrar en tablas y } \\
\text { gráficos de barra simple, } \\
\text { resultados de juegos } \\
\text { aleatorios con dados y } \\
\text { monedas. }\end{array}$ & $\begin{array}{l}\text { - Registran resultados de juegos aleatorios } \\
\text { con dados y monedas en tablas. } \\
\text { Registran resultados de juegos aleatorios } \\
\text { con dados y monedas en gráficos de } \\
\text { barra simple. }\end{array}$ \\
\hline
\end{tabular}

Fuente: Ministerio de Educación (2013a y b) 
En atención a estas consideraciones, nos plateamos el objetivo de investigar la habilidad de construcción de tablas estadísticas en estudiantes de tercer año de Educación Primaria en el contexto chileno.

En lo que sigue presentamos: el marco teórico donde mostramos los principales aspectos de las tablas estadísticas y sus tipos (fundamentos); los antecedentes de investigación, la metodología empleada, la descripción de los resultados y, finalmente las conclusiones.

\section{Fundamentación Teórica}

En los últimos años la investigación sobre el desarrollo de actividades con tablas estadísticas por parte estudiantes de primeros cursos de Educación Primaria poco a poco ha ido mostrado algunos avances. Lo que se ve motivado, principalmente, por la incorporación temprana de estas representaciones en la enseñanza.

Las tablas estadísticas, así como los gráficos, son consideradas "una de las modalidades específicas de registro y organización de la información útil cognitivamente para una multiplicidad de usos" (GABUCIO; MARTÍ; ENFEDAQUE; GILABERT; KONSTANTINIDOU, 2010, p. 184). Además, de un instrumento de transnumeración (WILD; PFANNKUCH, 1999), expresión que hace referencia a la nueva información que se obtiene al cambiar de registro de representación, por ejemplo, al pasar los datos no agrupados a una tabla de conteo se puede observar el dato de mayor frecuencia.

Estrella (2014) define tablas estadísticas como:

[...] un arreglo rectangular con una estructura que comprende un conjunto de filas y columnas [...], permite presentar los datos correspondientes a una o más variables (características del fenómeno bajo estudio) en forma clasificada y resumida, para permitir la visualización del comportamiento de los datos y facilitar la comprensión de la información que se puede extraer (p. 6).

Existen diferentes tipos de tablas estadísticas que se suelen trabajar en Educación Primaria y que movilizan diferentes elementos e ideas, estas son: 
- Tablas de datos. Corresponden a representaciones sencillas, en las que no se usa la idea de frecuencia ni de distribución, pero si las ideas de variable y valor.

- Tablas defrecuencias. Es aquella tabla en que se registran las frecuencias de aparición de una determinada categoría.

- Tablas de conteo. Es aquella en que se realiza un recuento de cada categoría en una determinada celda y cada valor de la variable se representa por medio de cierto símbolo; por lo que se puede considerar una simplificación de la tabla de frecuencia, ya que muchas veces este recuento va acompañado de este valor.

- Tabla de doble entrada. Es aquella que hace referencia a dos variables, una representada en las filas y otra en las columnas, es decir, un valor está relacionado con dos variables a la vez.

Dada la importancia de las tablas estadísticas a lo largo del currículo escolar, y sobre todo en los primeros cursos de Educación Primaria, surge la necesidad de investigaciones que se centren en este contenido, buscando verificar si son comprensibles para estudiantes de primeras edades educativas y profesores, estos últimos quienes deben enseñar esta temática en las aulas. Sin embargo, sigue siendo un tema que se encuentra en un estado incipiente y que requiere de más estudios. En lo que sigue se describen algunos de los estudios relacionados con esta temática.

Amorim y Silva (2016) analizaron actividades sobre tablas en cuatro libros de texto (dos de cuarto y dos de quinto) de Educación Primaria en Brasil. Los resultados muestran que las actividades centran su interés en la interpretación de la información, y que dichas tareas están relacionadas con situaciones cercanas a los estudiantes.

En el contexto chileno solo se encuentra el trabajo de Díaz-Levicoy, Morales y López-Martín (2015) quienes estudian las actividades sobre tablas estadísticas en cuatro libros de texto de Educación Primaria (dos de primero y dos de segundo). Los resultados 
evidencian el predominio de las tablas, donde las actividades más frecuentes son las de calcular y completar, es decir, aquellas en que se debe realizar alguna operación aritmética o comparación con los datos y en las que se pide finalizar la construcción de una tabla.

Respecto a la lectura de tablas, Espinoza (2015) desarrolla una experiencia de aula con 15 estudiantes de $1^{\circ}$ de Educación Primaria en Perú. Los resultados muestran que los estudiantes pueden realizar lectura de aspectos puntuales de las tablas estadísticas y pueden responder cuestiones relativas a las cantidades correspondientes de cada una de las categorías presentada en ella.

Por su parte, Ochoa (2015) realiza una experiencia de aula con estudiantes entre 7 y 8 años de edad en Perú sobre completar e interpretar tablas de doble entrada. Los resultados muestran que los estudiantes son capaces realizar con éxito la lectura puntual de tablas, y con dificultad actividades de cálculo.

Dada la importancia de los pictogramas en las directrices curriculares de diferentes países latinoamericanos (DÍAZ-LEVICOY; ARTEAGA; LÓPEZ-MARTÍN, 2015), recientemente, Díaz-Levicoy, Arteaga y Batanero (2017) estudian la compresión que alcanzan sobre la lectura de pictogramas en 380 estudiantes de $6^{\circ}$ de Educación Primaria de Chile. Una de las actividades consideradas en el instrumento pedía completar una tabla con la información de un pictograma de iconos no unitarios (cada icono representa 10 unidades estadísticas y la mitad 5). Los resultados permiten observar que el 74,4\% de los estudiantes completa correctamente la tabla, mientras que este porcentaje llega al 95,2\% si se consideran aquellas respuestas parcialmente correcta; por lo que los autores consideran una actividad sencilla para los estudiantes que están próximos a finalizar la Educación Primaria.

Desde esta perspectiva, con este trabajo, se busca aportar resultados novedosos en el contexto chileno, en el que la inclusión de los temas de estadística y probabilidad se ha introducido hace un par de años en la Educación Primaria, y donde el estudio aún es escaso. 


\section{Metodología}

En esta investigación seguimos una metodología cualitativa (PÉREZ-SERRANO, 1994), de tipo descriptivo (HERNÁNDEZ; FERNÁNDEZ; BAPTISTA, 2010). Para dar cuenta del objetivo de este estudio, se aplicó a 79 estudiantes de tres centros educativos $(25,27$ y 27 estudiantes por centro) un instrumento que construido de acuerdo con los resultados de un análisis sobre el tratamiento de este tema en libros de texto de $1^{\circ}$ y $2^{\circ}$ de Educación Primaria (DÍAZ-LEVICOY; MORALES; LÓPEZ-MARTÍN, 2015) y validado por juicio de expertos (DÍAZ-LEVICOY; MORALES; CRUZ; LÓPEZ-MARTÍN, 2016), a partir de dichas respuestas se realizó un análisis de contenido (LÓPEZ-NOGUERO, 2002). Se consideraron estudiantes de tercer curso porque de acuerdo a las directrices curriculares estos ya contarían con los conocimientos sobre tablas estadísticas descritas en las directrices curriculares.

El cuestionario constó de dos ítems en que los estudiantes debían construir una tabla con la estructura dada (Figura 2), esto ya que no se encontraron actividades de construcción sin este soporte (DÍAZ-LEVICOY; MORALES; LÓPEZ-MARTÍN, 2015).

\section{Resultados}

Las respuestas de los estudiantes ( $\mathrm{E} x$, donde $x$ representa su número del estudiante) fueron clasificadas de acuerdo a su calidad en: correctas, parcialmente correctas e incorrectas. En los que sigue explicamos el criterio utilizado en cada actividad, y por tipo de respuesta. Finalizamos con el estudio de la puntuación global.

La primera actividad (ver figura 2) pide completar una tabla de conteo a partir de un conjunto de datos sin agrupar, relacionada con el color favorito de los estudiantes de un $3^{\circ}$ de Educación Primaria. De esta actividad obtuvimos las siguientes categorías de respuestas:

Construcción correcta. Cuando el estudiante realiza correctamente el conteo y recuento de cada color que prefieren los estudiantes. Ejemplo de este tipo de repuesta es la de E26 (Figura 3), 
ACTIVIDAD 1. Lee la siguiente situación y desarrolla la actividad.

A cada uno de los estudiantes de $3^{\circ}$ básico se les preguntó por su color favorito. Las respuestas de cada uno de ellos fueron las siguientes:

rojo, azul, verde, rojo, amarillo, rojo, verde, verde, verde, azul, amarillo, rojo, rojo, verde, azul, azul, azul, verde, rojo, amarillo, azul, azul, amarillo, rojo, rojo

Completa la siguiente tabla con la información anterior

\begin{tabular}{|c|c|c|}
\hline \multicolumn{3}{|c|}{ COLOR PREFERIDO } \\
\hline Color & Conteo & Cantidad \\
\hline Rojo & & \\
\hline Azul & & \\
\hline Amarillo & & \\
\hline Verde & & \\
\hline
\end{tabular}

ACTIVIDAD 2. Lee la siguiente situación y desarrolla la actividad.

A un grupo de estudiantes de tercer año básico se les realizó una pregunta sobre su fruta preferida cuya información se organizó en el siguiente pictograma. Cada círculo representa el voto de dos estudiantes ( $0: 2$ estudiantes).

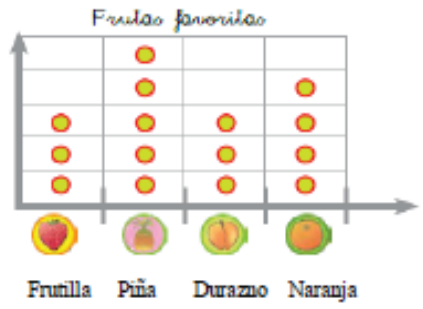

Completa la tabla

\begin{tabular}{|l|l|}
\hline \multicolumn{2}{|c|}{ Frutas favoritas } \\
\hline Tipo Fruta & Cantidad de votos \\
\hline Frutilla & \\
\hline Piña & \\
\hline Durazno & \\
\hline Naranja & \\
\hline
\end{tabular}


quien realiza correctamente el conteo por medio de segmentos verticales y su posterior recuento.

Figura 3. Construcción correcta (E26)

\begin{tabular}{|c|c|c|}
\hline \multicolumn{3}{|c|}{$\begin{array}{l}\text { COLOR PREFERIDO } \\
\end{array}$} \\
\hline Color & Conteo & Cantidad \\
\hline Rojo & 811111611 & 8 \\
\hline Azul & $715 i 111$ & 7 \\
\hline Amarillo & $4(11)$ & 4 \\
\hline Verde & 801111 & 6 \\
\hline
\end{tabular}

Construcción parcialmente correcta. Dentro de esta categoría incluimos aquellas respuestas en las que se observa un error de conteo (registrar o marcar el color favorito) o de recuento de los mismos. Si bien no se trata de un error relacionado con los convenios de los elementos de la tabla, se trata de la forma cuidadosa de registrar y contar. Ejemplo de este tipo de respuestas se muestra en la Figura 4, en la que E34 contabiliza mal la cantidad de estudiantes que prefieren el color verde, donde debería ser 6 y no 5. Además, observamos un error en el numeral utilizado para representar el cinco.

Figura 4. Construcción parcialmente correcta (E34)

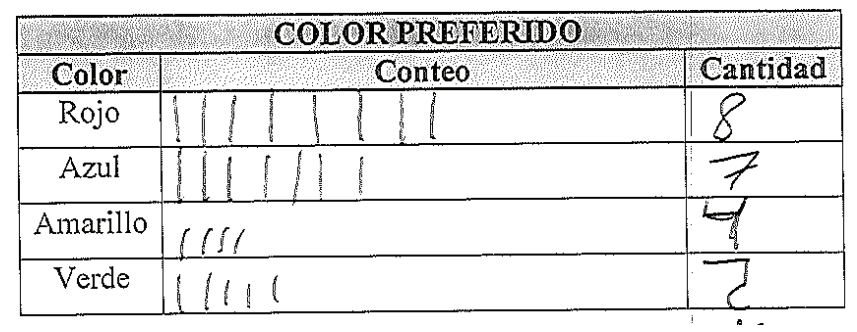

Construcción incorrecta. Se refiere a cuando el conteo y la frecuencia (cantidad) de la tabla construida no condicen con el listado de datos entregados en el enunciado de la actividad, también consideramos los casos en que los estudiantes no responden a la actividad. Por ejemplo, en esta categoría hemos incluido la respuesta 
de E27; construcción que se ha limitado a realizar el conteo solo de quienes prefieren el color rojo (que hace correctamente), pero cuando debe hacer el recuento se limita a escribir el número 1.

Figura 5. Construcción incorrecta (E27)

\begin{tabular}{|c|c|c|}
\hline \multicolumn{1}{|c|}{ COLOR ConERERDO } & \\
\hline Color & \multicolumn{1}{|c|}{ Conteo } & Cantidad \\
\hline Rojo & $1 /, 1 \mid 11 !$ & 1 \\
\hline Azul & & \\
\hline Amarillo & & \\
\hline Verde & & \\
\hline
\end{tabular}

La segunda actividad (ver figura 2) pide completar una tabla estadística a partir de la información proporcionada en un pictograma. Para responder correctamente a la actividad es necesario que se contabilice la cantidad de iconos y se relacione con su valor estadístico, en este caso la cantidad de iconos se debe multiplicar por 2. Las respuestas de los estudiantes se categorizaron siguiendo los siguientes criterios:

Construcción correcta. Ocurre cuando el estudiante lee correctamente la información del pictograma y lo registra en la tabla, ello implica leer la cantidad de iconos y multiplicar por 2, que corresponde al valor estadístico del icono. También se consideraron como correctas aquellas respuestas en cuyas tablas el estudiante realiza un conteo y considera que cada icono corresponde a dos unidades estadísticas. Por ejemplo, la respuesta de E74 (Figura 6) se basa en el conteo de los iconos (copia) y para obtener las cantidades multiplica por dos esas cantidades registradas. 
Figura 6. Construcción correcta (E74)

\begin{tabular}{|l|l|}
\hline \multicolumn{2}{|c|}{ Frutas favoritas } \\
\hline Tipo Fruta & Cantidad de votos \\
\hline Frutilla & $0 \bigcirc-6$ \\
\hline Piña & 000 \\
\hline Durazno & $0 \bigcirc-40$ \\
\hline Naranja & $\square Q 00-8$ \\
\hline
\end{tabular}

Parcialmente correcta. Corresponde a una respuesta que presenta algún error, ya sea el contar la cantidad de iconos o en confundir la categoría a la que corresponde un determinado valor. Pero, en general, el estudiante comprende los convenios de lectura de pictogramas y de construcción de tabla. En la Figura 7 vemos la respuesta de E77, en la que se registran y calculan correctamente tres de los cuatro valores, presentado error en la cantidad de Piñas, las que corresponde a 4 y no 6 .

Figura 7. Construcción parcialmente correcta (E77)

\begin{tabular}{|l|l|}
\hline \multicolumn{2}{|c|}{ Frutas favoritas } \\
\hline Tipo Fruta & Cantidad de votos \\
\hline Frutilla & \\
\hline Piña & \\
\hline Durazno & \\
\hline Naranja & \\
\hline
\end{tabular}

Incorrecta. Corresponde a aquella respuesta que se basa en la lectura literal del pictograma, es decir, se completa la tabla contabilizando solo la cantidad de iconos, sin considerar su valor estadístico. Otro tipo de respuesta que consideramos en esta categoría es aquella en que el estudiante considera valores diferentes y que no se 
corresponde con el valor de cada icono, o simplemente no responde a la actividad. En la Figura 8 vemos una respuesta considerada como incorrecta, ya que E65 se limita a realizar el conteo de iconos y no considera que su valor es diferente a la unidad. Este error es descrito en profundidad en Díaz-Levicoy, Arteaga y Batanero (2017) en su estudio sobre la compresión de pictogramas.

Figura 8. Construcción incorrecta (E65)

\begin{tabular}{|l|l|}
\hline \multicolumn{3}{|c|}{ Frutas favoritas } \\
\hline Tipo Fruta & Cantidad de votos \\
\hline Frutilla & \\
\hline Piña & \\
\hline Durazno
\end{tabular}

En la Tabla 1 mostramos la distribución del tipo de construcción que realizan los estudiantes a los ítems considerados en este trabajo. En ella vemos que la primera actividad es contestada correctamente por $68,4 \%$, logrando un $92,5 \%$ considerando aquellas respuestas parcialmente correctas. Mientras que en la segunda tarea, se observa que las respuestas correctas no superan el 50\% $(48,1 \%)$, $\mathrm{y}$ donde las respuestas incorrectas alcanzan un porcentaje semejante $(46,8 \%)$. Estos resultados evidencian que la segunda actividad presenta mayor dificultad para los estudiantes considerados en el estudio.

Tabla 1. Distribución de la frecuencia (porcentaje) del tipo de respuestas a los items 1 y 2

\begin{tabular}{ccc}
\hline Construcción & Ítem 1 & Ítem 2 \\
\hline Correctas & $54(68,4)$ & $38(48,1)$ \\
Parcialmente correctas & $19(24,1)$ & $4(5,1)$ \\
Incorrectas & $6(7,6)$ & $37(46,8)$ \\
\hline Total & $79(100)$ & $79(100)$ \\
\hline
\end{tabular}


Para realizar una valoración global de las respuestas de los estudiantes hemos asignado una determinada puntuación según la cantidad de sus respuestas (2: construcción correcta; 1: construcción parcialmente correcta; 0: construcción incorrecta).

En la Figura 9 observamos la distribución de los puntajes totales de los estudiantes mediante dos representaciones. La primera de ellas, mediante el gráfico de barras simples, muestra que distribución general de los puntajes obtenidos, en la que se vemos que una mayoría relativa de los estudiantes obtienen el máximo de la puntuación posible, es decir, realizan ambas construcciones correctamente. En la segunda gráfica (gráficos de barras adosadas) se muestra la distribución de los puntajes por la edad de los estudiantes (quitando los dos casos en que no se indicó), en dicho gráfico observamos que quienes tienen 8 años concentran el mayor porcentaje de la muestra y alcanzan los puntajes más altos (4 y 3 ).

Figura 9. Distribución de la puntuación total de los estudiantes
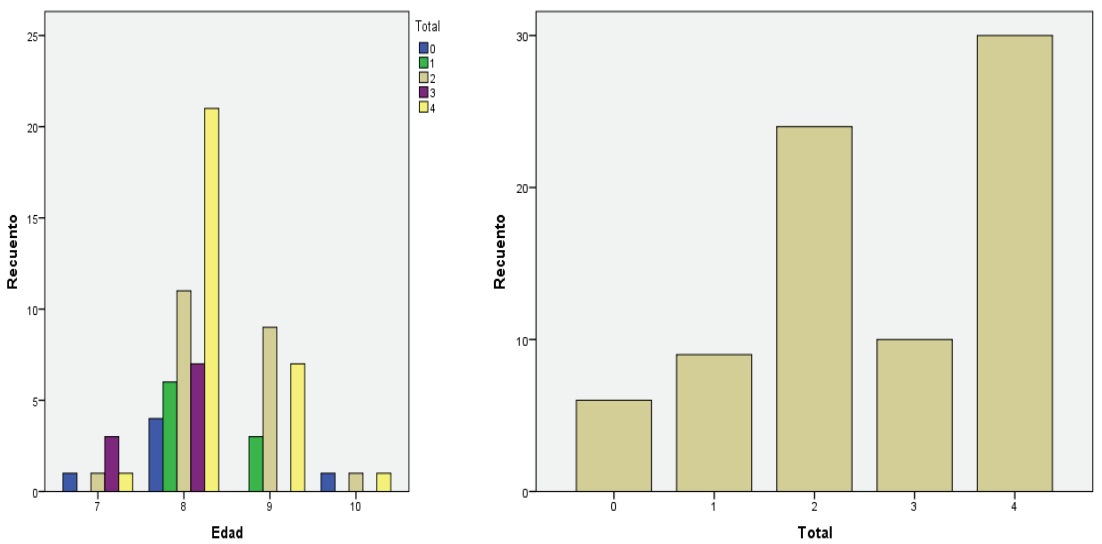


\section{Discusión y conclusión}

El incluir los temas de estadística y probabilidad en las actuales directrices curriculares chilenas de Educación Primaria tiene un gran impacto en el sistema educativo, por lo que estos temas deben abordarse en la formación inicial y continua de los profesores y en los libros de texto, para asegurar que estos temas sean comprendidos por los estudiantes.

En nuestro caso hemos indagado si los estudiantes de tercer año de Educación Primaria pueden construir tablas estadísticas, ya que se trata de un tema que se propone su enseñanza en los libros de texto de los dos primeros cursos de Educación Primaria (DÍAZ -LEVICOY; MORALES, LÓPEZ-MARTÍN, 2015).

Los resultados muestran que la primera actividad presentada (Figura 2) fue de mayor facilidad para los estudiantes, pues un $92,5 \%$ de los estudiantes realiza una construcción adecuada de la tabla (considerando las respuestas correctas y parcialmente correctas). Mientras que en la segunda actividad solo responder de esta forma el 53,2\%, y donde hay un alto porcentaje de respuestas incorrectas. A raíz de este resultado se podría sugerir que la actividad de transitar entre estos dos registros, es decir, desde la información de un pictograma a una tabla es más difícil que la actividad en la que debe realizar en conteo y obtener las frecuencias para estudiantes considerados en este estudio. Lo anterior, sucede en gran medida porque los estudiantes se limitan a contar la cantidad de iconos y no la relacionan con su valor estadístico. Este error también se ha observado en la investigación con estudiantes de $6^{\circ}$ de Educación Primaria de Chile (DÍAZ-LEVICOY; ARTEAGA; BATANERO, 2017). Los resultados (nivel de éxito) que hemos obtenido en el presente estudio son más bajos que los del estudio recientemente citado, lo que se puede justificar por la edad de los estudiantes ya que han trabajo con mayor variedad de tablas y gráficos estadísticos.

Los resultados muestran que los estudiantes trabajan y consideran aspectos de lectura literal de información, alcanzando con facilidad por estudiantes de primeros cursos de Educación Prima- 
ria, tal como lo confirman las investigaciones previas (e.g., ESPINOZA, 2015; GABUCIO et al., 2010; OCHOA, 2015; PEREIRA; CONTI, 2012).

A nivel general, se puede confirmar, a partir de las respuestas de los estudiantes, que las tablas estadísticas es un tema cuya enseñanza se aborda en las aulas. Sin embargo, creemos que se debe prestar mayor atención a aquellas actividades en que se pide traducir información de un gráfico estadístico a una tabla (cambio de registro). Especialmente, se debe poner atención en cada elemento y etiqueta de las representaciones involucradas, como el rótulo en que se indica el valor estadístico de cada icono, ya que aparentemente en el nivel que se hizo la investigación esto representa una dificultad para los estudiantes.

Como continuidad a este estudio creemos necesario ampliar la muestra, y de esta manera se podrían obtener resultados que puedan representar de mejor forma la realidad de los estudiantes chilenos, así como considerar otras habilidades como lectura de tablas estadísticas con sus diferentes niveles de complejidad.

\section{Referencias}

AMORIM, Natália, SILVA, Regina de Lima Silva. Apresentação e utilização de tabelas em livros didáticos de matemática do $4^{\circ}$ e $5^{\circ}$ anos do ensino fundamental. EM TEIA - Revista de Educação Matemática e Tecnológica Iberoamericana, Recife, v.7, n. 1, p. 1-21, Ene-Abr., 2016.

BATANERO, Carmen. Los retos de la cultura estadística. Yupana, Santa Fe, v. 1, n. 1, p. 27-37, 2004.

CAZORLA, Irene, UTSUMI, Miriam Cardoso. Reflexões sobre o ensino de estatística na educação básica. In: CAZORLA, Irene, SANTANA, Eurivalda (Coords.). Do tratamento da informação ao letramento estatístico. Itabuna: Via Litterarum, 2010.

DÍAZ-LEVICOY, Danilo, ARTEAGA, Pedro, BATANERO, Carmen. Lectura de pictogramas por estudiantes chilenos de Educación Primaria. In: MUNOZZ-ESCOLANO, José María, ARNAL-BAILERA, Alberto, BELTRÁN-PELLICER, Pablo, CALLEJO, María Luz, CARRILLO, José (Coords.). Investigación en Educación Matemática XXI. Zaragoza: SEIEM, 2017. 
DíAZ-LEVICOY, Danilo, ARTEAgA, Pedro, LÓPEZ-MARTín, María del Mar. Pictogramas en una muestra de directrices curriculares latinoamericanas. In: VÁSQUEZ, Claudia, RIVAS, Hernán, PINCHEIRA, Nataly, ROJAS, Francisco, SOLAR, Horacio, CHANDÍA, Eugenio, PARRAGUEZ, Marcela (Coords.), XIX Jornadas Nacionales de Educación Matemática. Villarrica: SOCHIEM, 2015.

DÍAZ-LEVICOY, Danilo, MORALES, Rodolfo, CRUZ, Andrea, LÓPEZ-MARTÍN, María del Mar. Validación de un cuestionario para evaluar la comprensión sobre tablas estadísticas en Educación Primaria. In: ESCARBAJAL, Andrés (Coord.). Libro de actas del IV Congreso Internacional de Investigación e Innovación en Educación Infantil y Educación Primaria. Murcia: Universidad de Murcia, 2016

DÍAZ-LEVICOY, Danilo, MORALES, Rodolfo, LÓPEZ-MARTÍN, María del Mar. Tablas estadísticas en libros de texto chilenos de $1^{\circ}$ y $2^{\circ}$ año de Educación Primaria. Revista Paranaense de Educação Matemática, Campo Mourão, v. 4, n. 7, p. 10-39, Jul-Dic., 2015.

ESPINOZA, Norma. Tablas y gráficos de barras a través del ciclo del pensamiento estadístico: un estudio con alumnos de primer grado de Educación Primaria. San Miguel, 2015, 92p. (Tesis de Magíster) - Pontificia Universidad Católica del Perú.

ESTRELLA, Soledad. El formato tabular: una revisión de literatura. Revista Actualidades Investigativas en Educación, San José, v. 14, n. 2, p. 1-23, May-Ago., 2014.

GABUCIO, Fernando, MARTÍ, Eduardo, ENFEDAQUE, Jesús, GILABERT, Sandra, KONSTANTINIDOU, Aikaterini. Niveles de comprensión de las tablas en alumnos de primaria y secundaria. Cultura y Educación, Madrid, v. 22, n. 2, p. 183-197, Abr-Jun., 2010.

GAL, Iddo. Adult's statistical literacy: Meaning, components, responsibilities. International Statistical Review, Voorburg, v. 70, n. 1, p. 1-25, Ene-Abr., 2002.

HERNÁNDEZ, Roberto, FERNÁNDEZ, Carlos, BAPTISTA, María del Pilar. Metodología de la investigación. 5ta. Ed. México: McGraw Hill, 2010.

LÓPEZ-NOGUERO, Fernando. El análisis de contenido como método de investigación. XXI. Revista de Educación, Huelva, v. 4, p. 167-180, 2002.

MINISTERIO DE EDUCACIÓN. Matemática educación básica. Bases curriculares. Santiago: Unidad de Currículum y Evaluación, 2012. 
MINISTERIO DE EDUCACIÓN. Matemática. Programa de estudio primer año básico. Santiago: Unidad de Currículum y Evaluación, 2013a.

MINISTERIO DE EDUCACIÓN. Matemática. Programa de estudio segundo año básico. Santiago: Unidad de Currículum y Evaluación, 2013b.

MURRAY, Scott, GAL, Iddo. Preparing for diversity in statistics literacy: Institutional and educational implications. In: PHILLIPS, Brian (Coord.). Proceedings of the Sixth International Conference on Teaching of Statistics. Ciudad del Cabo: IASE, 2002.

OCHOA, Edith del Rocío. El ciclo de investigación del pensamiento estadístico relacionado con tablas de doble entrada. Un estudio con alumnos del segundo grado de Educación Primaria. San Miguel, 2015, 113p. (Tesis de Magíster) - Pontificia Universidad Católica del Perú.

PEREIRA, Eduardo de Lucas, CONTI, Keli Cristina. Interpretando tabelas e construindo gráficos com alunos do $3^{\circ}$ ano do Ensino Fundamental. In: TOMMASIELLO, Maria Guiomar Carneiro, MARIN, Alda Junqueira, PIMENTA, Selma Garrido, CARVALHO, Luiz Marcelo, FUSARI, José Cerchi (Coords.). Didática e práticas de ensino na realidade escolar contemporânea: constatações, análises e proposições. Araraquara: Junqueira \& Marin, 2012.

PÉREZ-SERRANO, Gloria. Investigación cualitativa. Retos e interrogantes. I. Métodos. Madrid: La Muralla, 1994.

VÁSQUEZ, Claudia. Surgimiento del lenguaje probabilístico en el aula de Educación Primaria. REnCiMa, Revista de Ensino de Ciências e Matemática, São Paulo, en prensa, 2018.

WALLMAN, Katherine. Enhancing statistical literacy: enriching our society. Journal of the American Statistical Association, Virginia, v. 88, n. 421, p. 1-8, EneMar., 1993.

WATSON, Jane. Assessing statistical literacy through the use of media surveys. In: GAL, Iddo, GARFIELD, Joan (Coords.). The assessment challenge in statistics education. Amsterdam: IOS Press, 1997.

WILD, Chris, PFANNKUCH, Maxine. Statistical thinking in empirical enquiry. International Statistical Review, Voorburg, v. 67, n. 3, p. 223-265, Sep-Dic., 1999. 